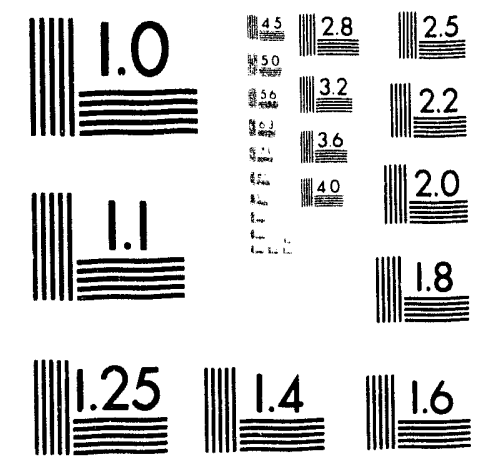



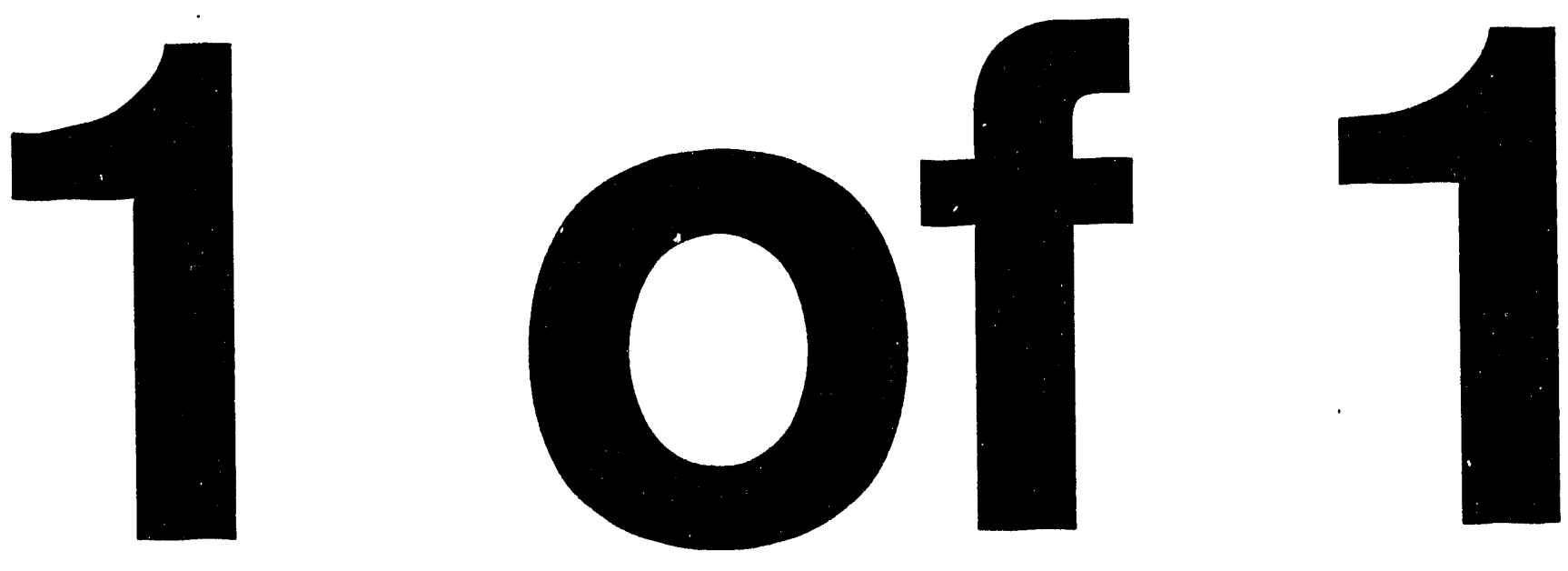
SANDIA REPORT

SAND 92-2744 $\cdot$ UL -405

Unlimited Release

Printed October 1993

Adaptive Path Planning in Changing Environments

Pang C. Chen

Prepared by

Sandra National Laboratories

Albuquerque, New Mexico 87185 and Livermore, California 94550

for the United States Department of Energy 
Issued by Sandia National Laboratories, uperated for the United States Department of Energy by Sandia Corporation.

NOTICE: This report was prepared as an account of work sponsored by an agency of the United States Government. Neither the United States Government nor any agency thereof, nor any of their employees, nor any of their contractors, subcontractors, or their employees, makes any warranty, express or implied, or assumes any legal liability or responsibility for the accuracy, completeness, or usefulness of any information, apparatus, product, or process disclosed, or represents that its use would not infringe privately owned rights. Reference herein to any specific commercial product, process, or service by trade name, trademark, manufacturer, or otherwise, does not necessarily constitute or imply its endorsement, recommendation, or favoring by the United States Government, any agency thereof or any of their contractors or subcontractors. The views and opinions expressed herein do not necessarily state or reflect those of the United States Government, any agency thereof or any of their contractors.

Printed in the United States of America. This report has been reproduced directly from the best available copy.

Available to DOE and DOE contractors from

Office of Scientific and Technical Information

PO Box 62

Oak Ridge, TN 37831

Prices available from (615) 576-8401, FTS 626-8401

Available to the public from

National Technical Information Service

US Department of Commerce

5285 Port Royal Rd

Springfield, VA 22161

NTIS price codes

Printed copy: A03

Microfiche copy: A01 


\title{
Adaptive Path Planning in Changing Environments*
}

\author{
Pang C. Chen \\ Sandia National Laboratories \\ Albuquerque, NM 87185
}

\begin{abstract}
Path planning needs to be fast to facilitate real-time robot programming. Unfortunately, current planning techniques are still too slow to be effective, as they often require several minutes, if not hours of computation. To overcome this difficulty, we present an adaptive algorithm that uses previous experience to speed up future performance. It is a learning algorithm suitable for incrementally-changing environments such as those encountered in manufacturing of evolving products and waste-site remediation. The algorithm extends our previous work for stationary environments in two directions: For minor environmental change, an object-attached experience abstraction scheme is introduced to increase the flexibility of the learned experience; for major environmental change, an on-demand experience repair scheme is also introduced to retain those experiences that remain valid and useful. In addition to presenting this algorithm, we identify three other variants with different repair strategies. To compare these algorithms, we develop an analytic model to compare the costs and benefits of the corresponding repair processes. Using this model, we formalize the concept of incremental change, and prove the optimality of our proposed algorithm under such change. Empirically, we also characterize the performance curve of each variant, confirm our theoretical optimality results, and demonstrate the practicality of our algorithm.
\end{abstract}

\section{Introduction}

In robotics, path planning refers to finding a short, collision-free path from an initial robot configuration to a desired configuration. It needs to be done quickly to support real-time task-level robot programming. Accordingly, it has received much attention [4], and there are now a number of implemented path planners based on a variety of approaches. Unfortunately, current planning techniques are still too slow to be effective, as they often require several minutes, if not hours of computation. To remedy this situation, we have previously presented an adaptive algorithm that uses past experience to increase future performance [2]. The algorithm is motivated by problems in radiation survey [3], and its effectiveness has been demonstrated both analytically and empirically. However, as stated, it is only applicable in stationary environments.

In this paper, we extend our algorithm and analysis to accommodate incrementally-changing environments. These environments are very common, with manufacturing of evolving products and waste-site remediation being potential applications. We extend the algorithm with two experiencemanipulating schemes designed to cope with minor and major environmental change. In addition

-This work has been performed at Sandia National Laboratories and supported by the U.S. Department of Energy under Contract DE-AC04-76DP00789. 
to presenting the algorithm, we also identify three other variant strategies for using old experiences in new environments.

In the following section, we will first quickly review our previous work on stationary environments, and then present the new algorithm for incrementally-changing environments. To gain insight into this algorithm, we will develop models and analyze the performance of the improved path planner in Section 4. Here, we shall formalize our notion of incremental change, and prove the optimality of our proposed algorithm under such change. Finally, in Section 5, we will demonstrate the effectiveness of the improved path planner in practice.

\section{Algorithm}

Given an arbitrary work environment and an arbitrary task $(u, u)$ of moving the robot from configuration point $u$ to $w$, we assume that there are initially two path planners available: Reach and Solve. The Reach planner is required to be fast, symmetric, and only locally effective, i.e., it should have a good chance of success if $u$ and $w$ are close to each other. The Solve planner, on the other hand, is required to be much more globally effective than Reach, and hence can be very slow. It is the performance of this planner that we wish to improve.

In our learning scheme, we retain the global effectiveness of Solve by calling it whenever necessary, while reducing the overall time cost by calling Reach whenever possible. To utilize Reach fruitfully, we maintain a history of robot movements in the form of a connected graph, called the experience graph $G=(V, E)$ with vertices $V$ and edges $E$. Set $V$ is a sparse collection of subgoals that the robot can attain and use. Set $E$ indicates the subgoal connections that the robot can follow through the application of Reach. Ideally, $G$ is to be used by Reach to achieve most tasks without the help of Solve. We update $G$ incrementally whenever Reach is incapable of achieving a task through $G$. In this situation, Solve is called. If Solve is also incapable of finding a solution, then we simply skip to the next task. Otherwise, the solution provided by Solve is abstracted (or compressed) into a chain consisting of a short sequence of subgoals that Reach can use later to achieve the same task. To learn from this experience, we simply generalize the use of the abstracted subgoals by augmenting $G$ with the chain.

\subsection{Environmental Assumptions}

We use the term 'home' to refer to the robot's home position if there is one, or the current position if there is none. To prevent the robot from losing track of home, we only allow environment changes that do not at anytime prevent the robot from moving back to its home easily (through $G$ with Reach). For applications in structured environments such as manufacturing, this requirement can be met by always having the robot back at home before introducing any workcell change. In less structured environments such as a waste site, we simply require that the robot not perform tasks to trap itself.

To allow fruitful learning, we also assume that the environmental change be incremental, i.e., occasional and localized. By occasional, we mean that the interval between workcell changes is large compared to the amount of time spent on each task. By localized, we mean that the workcell change involves only a few objects in a relative small area of the workspace, and hence is not extensive. Both conditions are prevalent in applications and have their intuitive implications: Occasional 


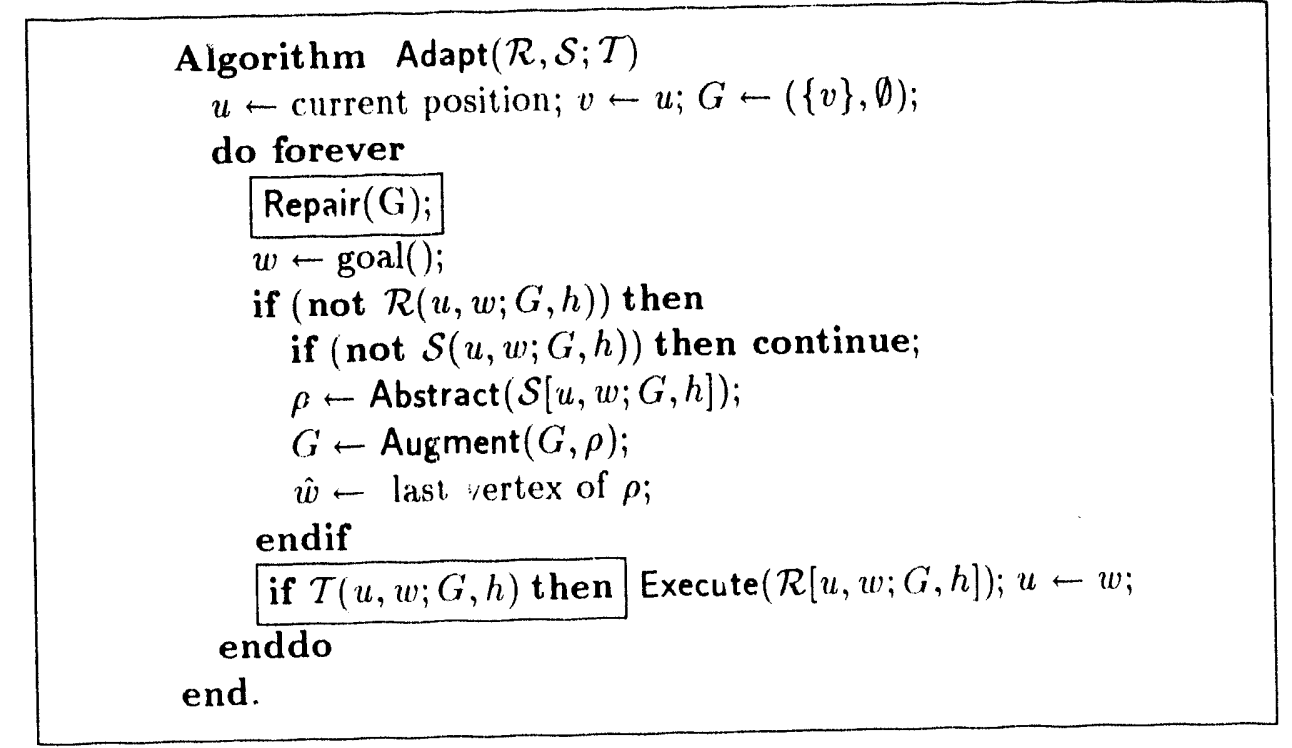

Figure 1: A learning algorithm for path planning in incrementally-changing environment

implies that old experience may be useful for significant amount of time, and localized implies that old experience may have salvage value.

\subsection{Formal Specification}

Formally, the speedup learning algorithm Adapt is shown in Figure 1. It is same as our earlier one for stationary environments [2] except for the extra boxed fragments. The second boxed fragment introduces $\mathcal{T}$, the trace procedure that verifies and repairs old experience on demand. The first fragment, which introduces Repair, is not part of the algorithm, but is included for later discussion of other variants of the algorithm (described in Section 2.5) that use it.

In the algorithm, $u$ is the current robot configuration, and $w$ is the next goal configuration. To access $G$, we maintain two pointers: $\hat{u}$ and $\hat{w}$, each of which points to a vertex of $G$ that is known to be reachable with one call of Reach from $u$ and $w$, respectively. The algorithm is based on two planners: $\mathcal{R}$ and $\mathcal{S}$, which are in turn based on Reach and Solve, respectively. Both $\mathcal{R}$ and $\mathcal{S}$ have task $(u, w)$ as arguments, and graph $(r$ and a heuristic vertex ordering function $h$ as parameters. For plann i $\mathcal{R}$, we use $\mathcal{R}(\cdot)$ to denote the predicate that $\mathcal{R}$ is successful, and $\mathcal{R}[\cdot]$ to denote the path planned when $\mathcal{R}$ succeeds, and similarly for $\mathcal{S}$.

Planner $\mathcal{R}$ searches for ways to achieve task $(u, w)$ using only Reach and $G$ as guideline. The algorithm for $\mathcal{R}(\cdot)$ is the same as before:

1. Search the vertices of $G$ in order according to heuristic $h$, and find a vertex $v$ satisfying $\operatorname{Reach}(v, w)$.

2. If $v$ exists, then set $\hat{w} \leftarrow v$, and return success.

3. Else return failure.

However, to generate $\mathcal{R}[\cdot]$, we now require the success of $\mathcal{T}(\cdot)$, which guarantees that there is a connected sequence of vertices $\Gamma$ in $G$ from $\Gamma_{1}=\hat{u}$ to $\Gamma_{k}=\hat{w}$ for some $k \geq 1$. Once $\mathcal{T}(\cdot)$ succeeds, 
a simple solution for $\mathcal{R}[\cdot]$ would be the concatenation of $\operatorname{Reach}\left[\Gamma_{j}, \Gamma_{j+1}\right]$ for $j$ going from 0 to $k$. However, we can also improve the quality of this solution locally as we shall see in Section 2.6.

Planner $\mathcal{S}$ sets $\hat{w}$ for the future augmentation of $G$, and is unchanged from before [2]. To evaluate $\mathcal{S}(\cdot)$, the algorithm is:

1. Set $\hat{w}$ to be the best vertex in $G$ according to $h$.

2. Return(Solve $(\hat{w}, w))$.

To generate $\mathcal{S}[\cdot]$ once $\mathcal{S}(\cdot)$ returns success, simply output Solve $[\hat{w}, w]$.

\subsection{Object-Attached Experience Abstraction}

To abstract a solution path from $v$ to $w$ with $v \in G$, we again assume as in [2] that there is an efficient $\operatorname{Abstract}(\cdot)$ function available that returns a short chain from $v$ to $v^{\prime}=w$, traversable by Reach. We assume that the size of the chains abstracted from solutions of $\mathcal{S}$ are all boundable by a constant. In practice, this is a reasonable assumption, since a typical task consists of only 3 smooth motions: departure, traversal, and approach.

However, to increase the flexibility of the subgoals, we now require the vertices returned by Abstract $(\cdot)$ to be relative robot positions associated with nearby objects, rather than the absolute positions in the stationary case. That is, instead of remembering the robot positions as some points in absolute space, we now remember each of them as an offset from some nearby object serving as a landmark. Under this object-attached experience abstraction scheme, we can adjust to any minor environmental change without expensive experience repair. This power is further illustrated in Section 3.

\subsection{On-Demand Experience Repair}

Of course, if the environment changes significantly, the validity of $G$ will deteriorate. How much deterioration will $G$ suffer depends on how drastically the environment changes. If the change is major and extensive, then it may be better to start over with no experience ( $G$ reinitialized), rather than to work with the old impaired experience. In the more interesting case where the change may be major (e.g., introducing a new object) but not extensive (e.g., the rest of the workcell is undisturbed), the right choice is not as clear. Therefore, we introduce an on-demand repair scheme (second boxed fragment in Figure 1) to retain those experiences that remain valid and useful.

In this scheme, we plan as if $G$ is connected, until $\mathcal{R}(\cdot)$ succeeds and we actually need to produce a path. Then, to generate $\mathcal{R}[\cdot]$, we require the success of $\mathcal{T}(\cdot)$ to provide a connected sequence from $\hat{u}$ to $\hat{w}$. As $\mathcal{T}(\cdot)$ searches for and verifies such a sequence, it may come across invalid edges, which it simply deletes. If $\hat{u}$ is already connected to $\hat{w}$ in $G$, then no repair need take place. If, however, $\hat{u}$ and $\hat{w}$ do not belong to the same (connected) component due to the deterioration of $G$, then Solve is called to reestablish their connectivity. It is of course possible that connectivity cannot be reestablished due to the environmental change. In this case, the portion of $G$ connected to $\hat{w}$ is deemed useless, and hence discarded. The procedure for $\mathcal{T}(\cdot)$ is as follows:

1. While there exists a sequence $\Gamma$ of vertices in $G$ connecting $\hat{u}=\Gamma_{1}$ to $\hat{w}=\Gamma_{k}$ for some $k \geq 1$ do

(a) If Reach $\left(\Gamma_{i}, \Gamma_{i+1}\right)$ for all $1 \leq i<k$ then return success;

(b) Else remove edge $\left(\Gamma_{i}, \Gamma_{i+1}\right)$ with smallest $i$ such that $\neg \operatorname{Reach}\left(\Gamma_{i}, \Gamma_{i+1}\right)$. 
2. If Solve $(\hat{u}, \hat{w})$ then augment $G$ with Abstract(Solve $[\hat{u}, \hat{w}])$; return success;

3 . Else remove the (connected) component of $\hat{w}$ from $G$, and return failure.

\subsection{Other Repairing Strategies}

It is also possible to cope with major environmental change using other variants of the on-demand repairing strategy. One trivial strategy is simply to forget the old experience and start over (with ( $\rightarrow$ reinitialized) whenever there is a change in the environment. The corresponding algorithm, $\mathcal{A}_{0}$, can be obtained from Figure 1 by skipping the boxed condition, and defining $\operatorname{Repair}(G)$ to be the reinitialization procedure.

Another less trivial strategy is to verify each edge of $G$ first whenever there is a change. Then with the time investment, we can initialize $G$ to the home component. The corresponding algorithm, $\mathcal{A}_{1}$, can again be obtained from Figure 1 by skipping the boxed condition, and defining Repair $\left(G^{\prime}\right)$ to be the above home-component extraction procedure.

Notice that both strategies above oniy update $G$ according to environmental change, and do not really repair old experience. In contrast, a third strategy that repairs actively is to first apply $\mathcal{T}$ to attempt reaching every vertex of $G$ from home, before taking on any new task. The corresponding algorithm, $\mathcal{A}_{2}$, can be obtained from Figure 1 by skipping the boxed condition, and defining Repair( $C$ ) io be the above repair-all procedure.

All of the saggested algorithms (including the repair-on-demand algorithm $\mathcal{A}_{3}$ ) have their advantages and disadvantages. Intuitively, if the environment undergoes a major and extensive change, then starting over with $\mathcal{A}_{0}$ may be the best choice. On the other hand, if Solve costs much more than Reach, then using $\mathcal{A}_{1}$ to save some old experience may be better. Alternatively, if the change is only local, then repairing old experience with $\mathcal{A}_{2}$ or $\mathcal{A}_{3}$ may be more beneficial. Which algorithm to use thus depends on the particular application.

\subsection{Solution Quality and Redundancy}

So far we have focused on task solvability but not solution quality. If solution quality is not important, then in $\mathcal{R}[\cdot]$, we can simply produce the solution of going through I' with Reach. In this situation, the experience graph will always be a tree.

However, if solution quality is important, then it may be worthwhile to locally optimize $\Gamma$. A procedure for compressing a sequence $\left(\Gamma_{i}, \Gamma_{i+1}, \ldots, \Gamma_{k}\right)$ with $k>i+2$ is as follows:

1. $j-i+2$

2. while $j \leq k$ do

(a) while $j \leq k$ and $\operatorname{Reach}\left(\Gamma_{i}, \Gamma_{j}\right)$ do

i. auginent $G$ with edge $\left(\Gamma_{i}, \Gamma_{j}\right)$;

ii. $j-j+1$;

(b) mark $\left(\Gamma_{i}, \Gamma_{j}\right)$ unconnectable through Reach;

(c) $i-i+1$

(d) $\Gamma_{i}-\Gamma_{j-1}$

(e) $j-j+1$

The procedure seeks forward to "cut corners" whenever possible. 


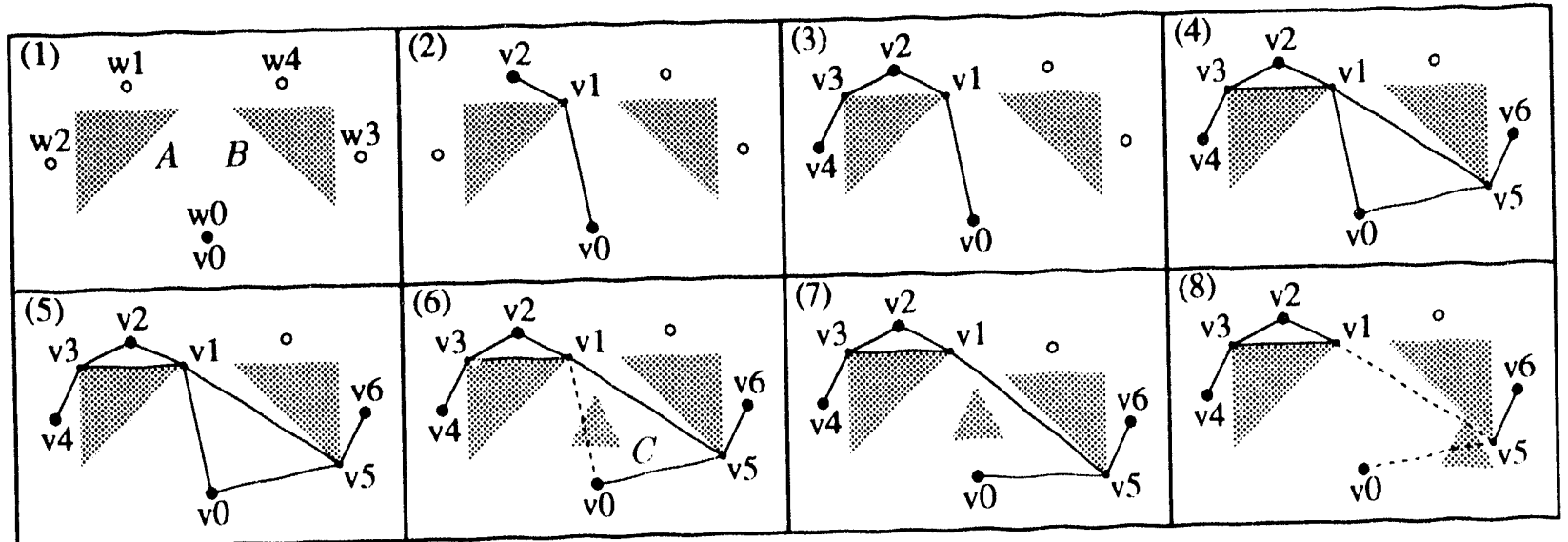

Figure 2: Snapshots of Adapt u1 der environmental change

In our work, we first use this procedure to compress the subsequence $\left(\Gamma_{1}, \ldots, \Gamma_{k}\right)$, which lies entirely in $G$, into a new sequence $\left(\Gamma_{1}, \ldots, \Gamma_{i}\right)$. The result of this compression is that $(i$ may be angmented with additional edges to enable shorter sequences in the future. Also, the redundancy introduced may be useful in combating against experience deterioration. To produce the actual sequence of subgoals for Reach to traverse, we run the compression procedure again on the new sequence with $u$ and $w$ attached to the ends as $\Gamma_{0}$ and $\Gamma_{i+1}$, respectively. This time, no graph augmentation need be done because $u$ and $w$ are not part of $G$.

\section{Example}

We illustrate the learning algorithm with a simple example involving a point robot in a 2l) workspace. (Similarly, the algorithm can plan for arbitrarily shaped and jointed robots by planning for a point robot in the configuration space.) Let Reach implement a go-straight procedure, with Reach $(u, w)$ returning success iff $u$ is visible from $u$, and Reach $[u, w]$ returning the line segment $\overline{u w}$. Let Solve implement a greedy 2 -step go-straight procedure, with Solve $(u, u)$ returning success iff the two points are connectable by at most 2 line segments, and Solve[ $u, w]$ returning the shortest such connecting path. To complete the algorithmic specification, let the heuristic used in $\mathcal{R}$ and $\mathcal{S}$ be $h=h_{1}$, with $h_{1}$ ordering the vertices of $G$ according to the distance to $w$, starting with the closest point first.

Figure 2 illustrates Adapt with a series of snapshots. Frame (1) shows the initial setting with the robot at home $u=w_{0}$ amongst two objects $A$ and $B$. The robot's initial tasks are to inspect both $A$ from $w_{1}$ and $w_{2}$, and $B$ from $w_{3}$ and $w_{4}$. To begin, the experience graph $G$ is initialized to the single vertex $v_{0}=w_{0}$.

The first goal indicated by $w_{1}$ is shown in Frame (2). Since $\mathcal{R}$ is unable to plan using only Reach and $G$, Adapt then calls $\mathcal{S}$. Using $h, \mathcal{S}$ chooses to extend from $v_{0}$ to $w_{1}$, since $v_{0}$ is the only vertex in $G$. The path produced by Solve $\left(v_{0}, w_{1}\right)$ consists of the line segments $\overline{v_{0} v_{1}}$ and $\overline{v_{1} v_{2}}$. This path is then abstracted into the chain connecting $v_{0}$ to $v_{1}$ and $v_{1}$ to $v_{2}$. The result of augmenting $G$ is that $G$ now becomes the 3 -vertex chain. Using this augmented $G, \mathcal{R}$ is now able to produce a path from $u=w_{0}$ to $w_{1}$, which consists of the segments $\overline{u v_{0}}, \overline{v_{0} v_{1}}, \overline{v_{1} v_{2}}$, and $\overline{v_{2} w_{1}}$, with $\overline{u v_{0}}$ and $\overline{v_{2} w_{1}}$ being null segments. 
With the first task accomplished, the next task is to go to $w_{2}$ shown in Frame (3). Since $\mathcal{R}$ is again unable to plan using only Reach and $G$, Adapt then calls $\mathcal{S}$. Using $h, \mathcal{S}$ chooses to extend from $v_{2}$ to $w_{2}$, and produces the line segments $\overline{v_{2} v_{3}}$ and $\overline{v_{3} v_{4}}$. The result of augmenting $G$ is that $G$ now becomes the 5 -vertex chain with new vertices $v_{3}$ and $v_{4}$.

With this $G, \mathcal{R}$ is still unable to succeed in reaching $w_{3}$ in Frame (4). Consequently, $\mathcal{S}$ chooses to extend from $v_{0}$ and produces 2 more segments $\overline{v_{0} v_{5}}$ and $\overline{v_{5} v_{6}}$. Thus, before calling $\mathcal{R}[\cdot], G$ is a 7 -vertex chain with new vertices $v_{5}$ and $v_{6}$. After calling $\mathcal{R}[\cdot[\cdot]$, however, $G$ becomes cyclic due to the addition of edges $\left(v_{3}, v_{1}\right)$ and $\left(v_{1}, v_{5}\right)$ as a result of locally optimizing the solution path $\left(v_{4}, v_{3}, v_{2}, v_{1}, v_{0}, v_{5}, v_{6}\right)$.

Frame (5) shows that $\mathcal{R}$ is now capable of reaching $w_{4}$, with $\hat{w}_{4}=v_{1}$. Consequently, $\mathcal{S}$ is not called for the first time, and $G$ is not modified.

So far, the workcell has been stationary. In Frame (6), we return the robot to its home and introduce a new object $C$. With $\mathcal{A}_{0}$ using the start-over strategy, we would lose the entire $G$ and not retain anything from the 3 previous calls to $\mathcal{S}$. With $\mathcal{A}_{1}$, we would verify all 8 edges in $G$ with Reach, remove the only broken edge $\left(v_{0}, v_{1}\right)$, and retain the rest of $G$ since it remains connected. If Reach costs much less than Solve, then the return on the initial time investment is certainly justifiable compared to that of $\mathcal{A}_{0}$. This case demonstrates that improving solution quality can also increase experience redundancy, which in turn decreases experience deterioration under change. With $\mathcal{A}_{2}$ using the active-repair scheme, we would also just remove edge $\left(v_{0}, v_{1}\right)$ from $G$ at the end of Repair $(G)$. With $\mathcal{A}_{3}$ using the repair-on-demand strategy, we simply do nothing.

Frame (7) shows what happens if we introduce some minor change by moving object $B$ and its object-attached goals $w_{3}$ and $w_{4}$. Because of the object-attached abstraction scheme, $v_{5}$ and $v_{6}$ also move along with $B$. Consequently, if the robot were to go back to $w_{3}$, it would again succeed by simply reaching toward $v_{5}$ and $v_{6}$.

Frame (8) shows what happens if we move object $C$ to a corner and decide not to inspect object $B$ anymore. In this case, $\mathcal{A}_{1}$ would be identical to $\mathcal{A}_{0}$ in reducing $G$ back to the single vertex $v_{0}$, except that $\mathcal{A}_{1}$ would also have to spend time verifying all 7 edges of $G$ before removing them. With $\mathcal{A}_{2}, G$ would be actively repaired, which means that it would call Solve twice to reestablish the connectivity of the 2 components to $v_{0}$. With $\mathcal{A}_{3}$, we again do nothing until the need arises. If we choose not to inspect $B$ anymore, then only one component needs to be reconnected to $v_{0}$, which means only one additional call to Solve would be required in the future. This case demonstrates the situation where using $\mathcal{A}_{3}$ is better than using $\mathcal{A}_{2}$.

\section{Analysis}

To analyze our (experience repair) algorithms in more detail, we develop an analytic model that is simple enough for probabilistic treatment, yet general enough to capture the key aspects of the learning process. The development of the model is in the same spirit as that in developing search tree models for analyzing heuristics [6]. We make the simplistic assumption that Solve is complete (able to find a solution when there is one) and that every task is solvable. We also assume that only Solve and Reach have costs, each being a constant. Let $r$ (typically $\ll 1$ ) be the cost ratio of Reach to Solve. The following definition introduces the notion of repair cost and benefit, and provides a way to compare different repair algorithms. 
Definition 1 An experience repair process has two associated quantities: cost $C$ and bene fit $B$. The repair cost measures the amount of time required to ensure the validity of the mended experience. The repair benefit measures the amount of time required to reconstruct the mended experience from scratch. The value and efficiency of the repair process are defined as $B-C$ and $B / C$, respectively. For algorithm comparisons, we say that (on average) $\mathcal{A}$ dominates $\mathcal{A}^{\prime}$, denoted by $\mathcal{A} \succeq \mathcal{A}^{\prime}$, if the (average) difference between the repair value of $\mathcal{A}$ and that of $\mathcal{A}^{\prime}$ is nonnegative.

The notion of benefit reflects the principle of "no pain, no gain". Intuitively, if $\mathcal{A}$ dominates $\mathcal{A}^{\prime}$, then $\mathcal{A}$ is better than $\mathcal{A}^{\prime}$ in the sense that either more experience is produced or less training is required. Notice that occasional environment change is implicitly assumed here to allow "benefits" to be fully utilized as usefu! experience. 'To compare the algorithms in more detail, we introduce the following model of experience graph and measures of repair cost and benefit.

Definition 2 Let $\|X\|$ for any griph $X$ be the number of edges in $X$. Let $T_{0}$ be a one-vertex tref consisting of only the root (home) $v_{0}$. For $n>0, T_{n}$ is a random tree oblained from $T_{n-1}$ by extending from a uniformly chosen vertex of $T_{n-1}$ a branch $\rho_{n}$ consisting of an alternating sequence of edges and vertices. The length (number of edges) of $\rho_{n}$ is a random variable $\|\rho\| \geq 1$ that is independent of $n$.

Define the applicability of an subgraph $H \subseteq G$ to be the probability that $\mathcal{R}(\cdot)$ will succeed for the next random goal $w$ with $\hat{w} \in H$.

Under model $\mathcal{M}$, the following assumptions on the experience graph and its repair hold:

1. The experience graph is $G=T_{n}$ with $n$ indexing occurrences of learning.

2. Each edge has an independent probability $p=1-q$ of becoming invalid under an environment change.

3. The benefit of any subgraph $H \subseteq(i$ is the sum of the benefit of every valid edge of $H$, each modeled by an independent, identically distributed nonnegative random variable 1 with mean $\nu=1 / \mathbf{E}\|\rho\|$.

4. Benefit and applicability are positively correlated in that a portion of (: has a higher benefit than another iff it has a larger applicability, and that positive benefit implies positive. applirability and vice versa.

5. Experience repair constitutes no learning, i.e., any additional structure introdured during repair has no benefit.

Thus, each branch $\rho$ in $T_{n}$ corresponds to the abstracted solution of a call to Solve. The reason for $\ddot{\nu}=1 / \mathbf{E}\|\rho\|$ is based on the assumption that each branch costs 1 and has on average $\mathbf{E}\|\rho\|$ number of edges. 'The idea that more benefit means more useful experience is implicitly assumed through the positive correlation between benefit and applicability. Notice that $\mathcal{M}$ is designed to model Adapt without local optimization (Section 2.6), where the experience graph is always a tree.

We now use $\mathcal{M}$ to investigate the situations for which $\mathcal{A}_{3}$ is better than the other variants.

Theorem 1 Suppose that under $\mathcal{M}, T_{n}$ for some fixed $n$ is the experience before environment change. Then $\mathcal{A}_{2} \succeq \mathcal{A}_{0}$ on average over all $T_{n}$ if

$$
p \leq(\bar{\nu}-r) /(1+\bar{\nu})
$$

independent of $n$. 
Proof The average extra benefit saved by $\mathcal{A}_{2}$ over $\mathcal{A}_{0}$ is $\bar{\nu}$ times $q \mathbf{E}\left\|T_{n}\right\|$, the expected number of valid edges in $T_{n}$, averaged over all $T_{n}$. The average extra cost incurred by $\mathcal{A}_{2}$ over $\mathcal{A}_{0}$ in saving this benefit is at most $r$ times the average number of edges in $T_{n}$, which accounts for the verification efforts (calls to Reach) of each edge, plus the expected number of edge removals for trees, which accounts for the number of calls to Solve to reconnect. This extra cost is $r \mathbf{E}\left\|T_{n}\right\|+p \mathbf{E}\left\|T_{n}\right\|$. Therefore, for $\mathcal{A}_{2}$ to dominate $\mathcal{A}_{0}$ on average, it suffices to have $\bar{\nu} q \mathbf{E}\left\|T_{n}\right\| \geq(r+p) \mathbf{E}\left\|T_{n}\right\|$, or equivalently $p \leq(\bar{\nu}-r) /(1+\bar{\nu})$, to guarantee the nonnegativity of the difference in repair value.

Thus, if $r$ is not sufficiently small, i.e., less than $\bar{\nu}$, then starting over from scratch with $\mathcal{A}_{0}$ may be better than actively repairing with $\mathcal{A}_{2}$. Fortunately, $r$ is typically orders of magnitude smaller than 1 (thus, the need for improving Solve). To interpret the theorem more concretely, consider a typical case where $r=1 / 10$ and Abstract yields at most 3 pieces of paths (departure, traversal, approach), each corresponding to an edge. In this case, $\mathbf{E}\|\rho\| \leq 3$ implies that $\nu \geq 1 / 3$. Hence, by the theorem above, if the environment change is both occasional and local, having a sufficiently low rate of change $(p \leq(1 / 3-1 / 10) /(1+1 / 3)=17.5 \%)$ then $\mathcal{A}_{2}$ will be better than $\mathcal{A}_{0}$.

Lemma 2 Let each edge of $T_{n}$ have a probability $p$ of being removed, and let $f_{n}$ be the number of branches of $T_{n}$ having their first vertices unreachable from the root. Then the number of vertices of $T_{n}$ not reachcble from the root, averaged over all $T_{n}$, is at least $\mathbf{E} f_{n} \mathbf{E}\|\rho\|$, where

$$
\mathbf{E} f_{n} \geq p(n+1)^{q}(\ln (n+1)-1 / 2) .
$$

Proof Since the length of each branch is independent, the expected number of vertices of $T_{n}$ not reachable from the root is obviously bounded below by $\mathbf{E} f_{n} \mathbf{E}\|\rho\|$. To obtain the lower bound on $\mathbf{E} f_{n}$, let $T_{n}^{\prime}$ be the graph after each edge of $T_{n}$ is randomly removed with probability $p$. Let $\rho_{[i]}$ with $i$ going from 1 to $f_{n}$ be the $f_{n}$ branches of $T_{n}$ having their first vertices unreachable from the root. Since $T_{n+1}$ is obtained by extending $\rho_{n+1}$ from a uniformly chosen vertex $v$ of $T_{n}$, branch $\rho_{n+1}$ can be counted in $f_{n+1}$ by having either its first edge removed, or its first edge intact but $v$ in $\rho_{[i]}$ for some $i$. Hence, we have

$$
\mathbf{E}\left(f_{n+1} \mid T_{n}^{\prime}\right) \geq f_{n}+p+q \sum_{i=1}^{f_{n}}\left\|\rho_{[i]}\right\| /\left(1+\left\|T_{n}\right\|\right) .
$$

Since $\left\|\rho_{i}\right\| /\left(1+\left\|T_{n}\right\|\right)$ has the same distribution for all $i$, and $\sum_{i=1}^{n}\left\|\rho_{i}\right\|=\left\|T_{n}\right\|$, we must have $\mathbf{E}\left\|\rho_{i}\right\| /\left(1+\left\|T_{n}\right\|\right) \geq 1 /(n+1)$. Therefore,

$$
\begin{aligned}
\mathbf{E} f_{n+1} & \geq p+\mathbf{E} f_{n}+q \mathbf{E}\left(\mathbf{E}\left(\sum_{i=1}^{f_{n}}\left\|\rho_{[i]}\right\| /\left(1+\left\|T_{n}\right\|\right) \mid f_{n}\right)\right) \\
& \geq p+\mathbf{E} f_{n}+q \mathbf{E} f_{n} /(n+1) \\
& =p+(1+q /(n+1)) \mathbf{E} f_{n} .
\end{aligned}
$$

Unfolding the recurrence yields

$$
\mathbf{E} f_{n} \geq p \sum_{i=1}^{n} \prod_{j=i+1}^{n}\left(1+\frac{q}{j}\right)
$$




$$
\begin{aligned}
& =p \sum_{i=1}^{n} \prod_{j=i+1}^{n}\left(1-\frac{p}{j+1}\right) \frac{j+1}{j} \\
& =p \sum_{i=1}^{n} \frac{n+1}{i+1} \prod_{j=i+1}^{n}\left(1-\frac{p}{j+1}\right) \\
& \geq p \sum_{i=1}^{n} \frac{n+1}{i+1} \prod_{j=i+1}^{n}\left(1-\frac{1}{j+1}\right)^{p} \\
& \geq p \sum_{i=1}^{n} \frac{n+1}{i+1}\left(\frac{i+1}{n+1}\right)^{p} \\
& \geq p(n+1)^{q} \sum_{i=1}^{n} \frac{1}{i+1} \\
& \geq p(n+1)^{q}(\ln (n+1)-1 / 2) .
\end{aligned}
$$

Theorem 3 Suppose that under $\mathcal{M}, T_{n}$ for some fixed $n$ is the experience before environment change. Then $\mathcal{A}_{2} \succeq \mathcal{A}_{1}$ on average over all $T_{n}$ if

$$
p \leq \ln (\nu(\ln (n+1)-1 / 2)) / \ln n .
$$

Proof The average extra benefit saved by $\mathcal{A}_{2}$ over $\mathcal{A}_{1}$ is $\ddot{\nu}$ times the expected number of non-root vertices in $T_{n}$ not reachable from the root, or equivalently, the number of valid edges, averaged over all $T_{n}$. By Lemma 2, this extra benefit is at least $\nu p(n+1)^{q}(\ln (n+1)-1 / 2) \mathbf{E}\|\rho\|$. The average extra cost incurred by $\mathcal{A}_{2}$ over $\mathcal{A}_{1}$ in saving this benefit is the expected number of edge removals for trees, which accounts for the number of calls to Solve. This extra cost is $p \mathrm{E}\left\|T_{n}\right\|=p n \mathrm{E}\|\rho\|=p n / \nu$. Therefore, for $\mathcal{A}_{2}$ to dominate $\mathcal{A}_{1}$ on average, it suffices to have

$$
p(n+1)^{q}(\ln (n+1)-1 / 2) \geq p n / \ddot{\nu}
$$

to guarantee the nonnegativity of the difference in repair value. This inequality can be attained by having $(\ln (n+1)-1 / 2) \geq n^{p} / \ddot{\nu}$, which yields the theorem.

Thus, the acceptable probability of change for which $\mathcal{A}_{2} \succeq \mathcal{A}_{1}$ actually decreases (although slowly) as the amount of learned knowledge increases. An explanation for this phenomenon is that as $\boldsymbol{n}$ increases, the proportional amount of 'deep' versus 'shallow' knowledges decreases, which favors $\mathcal{A}_{1}$ over $\mathcal{A}_{2}$ because only 'deep' knowledge is typically worth repairing. To interpret the theorem mure concretely, consider again the typical case with $\nu \geq 1 / 3$. Let $n<1000$ be a bound on the amount of learning already accomplished. Then by the theorem above, if the environment change is both occasional and local with probability of change $p \leq \ln ((\ln 1001-1 / 2) / 3) / \ln 1000 \doteq 11 \%$, then $\mathcal{A}_{2}$ will be better than $\mathcal{A}_{1}$.

Lemma 4 Given a vector $\vec{p}$ with increasing probability components $p_{i}$, define vectors $\vec{x}_{\ell}$ and $\vec{y}_{\ell}$ for $\ell \geq 1$ with $x_{\ell, i}=p_{i} \sum_{j<\ell} q_{i}^{j} \cdot q_{i}=1-p_{i}$, and $y_{\ell, i}=x_{\ell, i} /\left(c+\sum_{i} x_{\ell, i}\right)$ for some $c \geq 0$. Then $\vec{y}_{\ell}$ majorizes $\vec{y}_{\ell+1}$ in that $\sum_{1 \leq k} y_{\ell, 1} \leq \sum_{1 \leq k} y_{\ell+1, i}$ for all $k$. 
Proof The condition for majorization is equivalent to

$$
\begin{aligned}
\sum_{i \leq k} x_{\ell, i}\left(c+\sum_{i} x_{\ell+1, i}\right) & \leq \sum_{i \leq k} x_{\ell+1, i}\left(c+\sum_{i} x_{\ell, i}\right) \\
\Longleftrightarrow \sum_{i \leq k} x_{\ell, i} \sum_{i} x_{\ell+1, i} & \leq \sum_{i \leq k} x_{\ell+1, i} \sum_{i} x_{\ell, i} \\
\Longleftrightarrow \quad \sum_{i \leq k} x_{\ell, i} \sum_{i} p_{i} q_{i}^{\ell} & \leq \sum_{i \leq k} p_{i} q_{i}^{\ell} \sum_{i} x_{\ell, i} \\
\Longleftrightarrow \quad \sum_{i \leq k} x_{\ell, i} \sum_{i>k} p_{i} q_{i}^{\ell} & \leq \sum_{i \leq k} p_{i} q_{i}^{\ell} \sum_{i>k} x_{\ell, i} .
\end{aligned}
$$

The last inequality holds if $x_{\ell, i} p_{i^{\prime}} q_{i^{\prime}}^{\ell} \leq p_{i} q_{i}^{\ell} x_{\ell, i^{\prime}}$ for $i<i^{\prime}$, which is equivalent to having $q^{\ell} / \sum_{j<\ell} q^{j}$ as a increasing function of $q$. Since the derivative of this function has the same sign as $\ell q^{\ell-1}-$ $(\ell+1) q^{\ell}+\ell q^{2 \ell}=\ell(1-p)^{\ell+1}+(\ell+1) p-1$, with $p=1-q$, it suffices to show the nonnegativity of the last function. Clearly, for $p \geq 1 /(\ell+1)$, the function is nonnegative. For $p \leq 1 /(\ell+1)$, $(1-p)^{\ell+1} \geq 1-(\ell+1) p \geq 0$. Hence the function is indeed nonnegative.

Theorem 5 On average under $\mathcal{M}$, the efficiency of algorithm $\mathcal{A}_{3}$ as a function of time is bounded below by a decreasing function with the efficiency of $\mathcal{A}_{2}$ being a lower bound.

Proof Let $G$ and $G^{\prime}$ be the respective experience graphs before and after environment change, and $\eta$ be the number of non-home components of $G^{\prime}$. Assume $\eta>0$, since otherwise, no repair cost is incurred, and the theorem becomes trivially true. Enumerate these components of $G^{\prime}$ in the order of increasing benefit. Let $\vec{\nu}$ and $\vec{p}$ be $\eta$-vectors with $\nu_{i}$ and $p_{i}=1-q_{i}$ being the benefit and the applicability of the $i^{\text {th }}$ component, respectively. From the positive correlation between benefit and applicability, we also have $\vec{p}$ increasing.

Let $\vec{x}_{\ell}$ be an $\eta$-vector with components $x_{\ell, i}=p_{i} \sum_{j<\ell} q_{i}^{j}$, and $\overrightarrow{1}$ be a $\eta$-vector of 1 's. Then after $\ell$ tasks in $\mathcal{A}_{3}$, graph component $i$ will, with probability $x_{\ell, i}$, be arplicable (through $\mathcal{R}(\cdot)$ ) and hence reconnected (through $\mathcal{T}(\cdot)$ and Solve) to the home-component. Consequently, the average benefit saved by $\mathcal{A}_{3}$ after $\ell$ tasks is $B_{\ell}=\vec{x}_{\ell} \cdot \vec{\nu}$, and the average cost incurred by $\mathcal{A}_{3}$ for this benefit is $C_{\ell} \leq \vec{x}_{\ell} \cdot \overrightarrow{1}+r\|G\|$.

Let $\vec{y}_{\ell}=\vec{x}_{\ell} /\left(\vec{x}_{\ell} \cdot \vec{I}+r\|G\|\right)$. Since $\vec{y}_{\ell}$ majorizes $\vec{y}_{\ell+1}$, and $\vec{\nu}$ is increasing, we have $\vec{y}_{\ell} \cdot \vec{\nu} \geq \vec{y}_{\ell+1} \cdot \vec{\nu}$ by a classical inequality [5, page 445]. Hence, the efficiency $B_{\ell} / C_{\ell}$ is bounded below by the decreasing function $\vec{y}_{\ell} \cdot \vec{\nu}$. Let $\eta^{\prime}$ be the number of nonzero $p_{i}$ 's. As $\ell$ approaches infinity, $W_{\ell} / E_{\ell}$ is bounded below by $\vec{\nu} \cdot \vec{l} /\left(\eta^{\prime}+r\|G\|\right)$, which is greater than or equal to $\vec{\nu} \cdot \overrightarrow{1} / \eta$, the efficiency of $\mathcal{A}_{2}$.

In other words, $\mathcal{A}_{3}$ always repairs more "efficiently" than $\mathcal{A}_{2}$. The intuition is that on-demand repair takes advantage of positive correlations between benefit and applicability, and consequently allows more useful components to be reconnected first.

Theorem 6 Let $G^{\prime}$ be the experience graph after environment change and $\nu_{1}$ be the smallest benefit of the non-home components of $G^{\prime}$. Then under $\mathcal{M}$, the expected difference between the repair value of $\mathcal{A}_{3}$ with sufficient training to that of $\mathcal{A}_{2}$ will either exceed 0 if $\nu_{1}<1$, or approach a nonnegative number otherwise. 


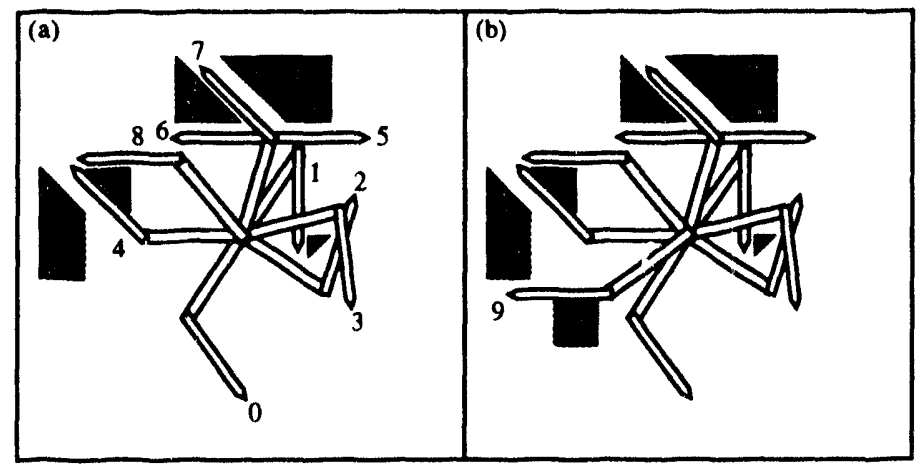

Figure 3: A planar 2-link robot environment with incremental change

Proof Continuing with the use of variables in the previous theorem, the repair value extracted by $\mathcal{A}_{2}$ is $\vec{\nu} \cdot \overrightarrow{1}-\eta-r\|G\|$ and that extracted by $\mathcal{A}_{3}$ is at least $\vec{x}_{\ell} \cdot \vec{\nu}-\vec{x}_{\ell} \cdot \overrightarrow{1}-r\|G\|$. Therefore, the value difference between that by $\mathcal{A}_{3}$ and $\mathcal{A}_{2}$ is at least $\left(\overrightarrow{1}-\vec{x}_{\ell}\right) \cdot(\overrightarrow{1}-\vec{\nu})$, which is equal to $\sum_{i=1}^{\eta} q_{i}^{\ell}\left(1-\nu_{i}\right)$. If $\nu_{1}<1$, then the first term will be positive and dominate the rest of the sum. If $\nu_{1} \geq 1$, then the sum will approach 0 from below.

Thus, we have shown that given any arbitrary $G$ and $G^{\prime}, \mathcal{A}_{3}$ will dominate $\mathcal{A}_{2}$ asymptotically (with sufficient training). To summarize, we have following corollary.

Corollary 7 Suppose that the environment change is incremental, i.e., occasional and local with sufficiently small rate of change indicated by

$$
p \leq \min ((\bar{\nu}-r) /(1+\bar{\nu}), \ln (\bar{\nu}(\ln (n+1)-1 / 2)) / \ln n)
$$

Then $\mathcal{A}_{3}$ is the best of the four variants in that it dominates asymptotically $\mathcal{A}_{2}$, which in turn dominates both $\mathcal{A}_{1}$ and $\mathcal{A}_{0}$.

\section{Computational Experience}

Using Adapt, we have again improved the performance of the path planner in [1], this time operating under environmental change. Figure 3 shows a 2 -link planar robot environment in which Adapt is applied. In this experiment, the initial environment shown in Frame (a) has 5 polygonal obstacles in the workcell and a goal set consisting of 9 preselected goal positions. Starting at home 0 , the robot is to go through a sequence of goals randomly selected from the goal set. During the exercise, we introduce an incremental environmental change, shown in Frame (b), by adding a new obstacle to the workcell and a new goal position to the goal set.

The result of this experiment, with Adapt using all 4 different repairing strategies, is shown in Figure 4. Here, the ratio of the cumulative planning cost required by Adapt to that required by Solve only is plotted against the task number. The planning costs are averaged over 100 runs and are measured by the number of robot-to-obstacle distance evaluations, which is the dominating factor in the computing cost of each planner. The environment change is introduced after task 40. To emphasize the important features of the result, the initial portion of the curve corresponding to ratios greater than 1 is not plotted. The unplotted portion actually decreases monotonically from 


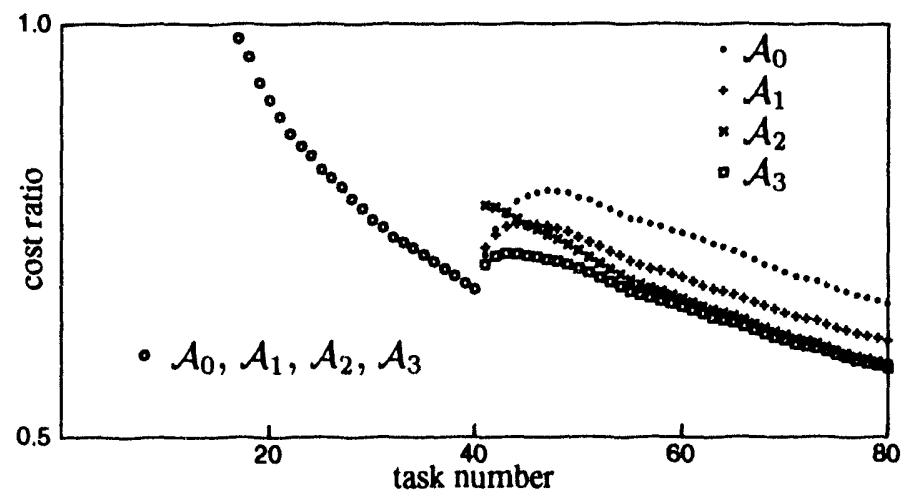

Figure 4: Time improvement of Adapt with all 4 repair strategies over Solve

2.5 at task number 1 to 1.0 at task number 16. The experiment shows that before the environmental change, Adapt is able to learn and speed up its performance relative to Solve from $150 \%$ slower to $33 \%$ faster. It also shows that Adapt needs about 16 training tasks before becoming competitive with Solve, a fact attributable to both the task simplicity for Solve and the significant costs incurred by Adapt during solution abstraction and compression.

After the environmental change, the performance curve for Adapt splits up into 4 curves, each corresponding to a different experience repairing strategy. The curves for $\mathcal{A}_{0}, \mathcal{A}_{1}$, and $\mathcal{A}_{3}$ exhibit similar behaviors in that they all gradually increase and then decrease at roughly the same rate, with $\mathcal{A}_{3}$ being clearly better than $\mathcal{A}_{1}$, which in turn being clearly better than $\mathcal{A}_{0}$. The curve for $\mathcal{A}_{2}$ is different in that it first jumps to a high point and then comes down rapidly to approach the curve for $\mathcal{A}_{3}$. The jump is due to the high initial cost of active repair, and the rapid decrease is due to the benefit of the repair. Overall, the relative performance of the repairing strategy is as expected, since the environmental change is incremental, involving only local and occasional change.

\section{Conclusion}

We have presented a speedup learning algorithm for path planning in incrementally changing environments. The algorithm extends our previous work for stationary environments with two augmenting experience-manipulating schemes: For minor environmental change, an object-attached experience abstraction scheme is introduced to increase the flexibility of the learned experience; for major environmental change, an on-demand experience repair scheme is introduced to retain those experiences that remain valid and useful.

In justifying our on-demand experience repair scheme $\left(\mathcal{A}_{3}\right)$, we have also identified three other variants with different repairing strategies: $\mathcal{A}_{0}$ simply forgets the old experience and starts over whenever there is a change; $\mathcal{A}_{1}$ first verifies the old experience and then retains only the home component; and $\mathcal{A}_{2}$ actively repairs the old experience before taking on new tasks. To compare these algorithms, we have developed an analytic model $\mathcal{M}$ to compare the costs and benefits of the corresponding repair processes. Using $\mathcal{M}$, we have formalized the concept of incremental change, and proved the optimality of $\mathcal{A}_{3}$ under such change.

Experimentally, we have also characterized the performance curve of each repairing scheme, and confirmed our theoretical results on the optimality of $\mathcal{A}_{3}$. In particular, we have demonstrated 
the practicality of our algorithm by improving the performance of an existing path planner under a changing environment.

\section{References}

[1] Chen, P.C., "Effective Path Planning through Task Restriction," Sandia Report SAND91-1964, 1992.

[2] Chen, P.C., "Improving Path Planning with Learning," Machine Learning: Proc. of the Ninth Ini. Conf., 1992.

[3] Harrigan, R.W., Sanders, T.L., "A Robotic System to Conduct Radiation and Contamination Surveys on Nuclear Waste Transport Casks," Sandia Report SAND89-0017, 1990.

[4] Latombe, J., Robot Motion Planning, Kluwer Academic. Publishers, 1991.

[5] Marshall, A.W., Olkin, I., Inequalities: Theory of Majorization and Its Applications, Academic Press, 1979.

[6] Pearl, J., Heuristics: Intelligent Search Strategies for Computer Problem Solving, AddisonWesley, 1984. 
Distribution:

7141 Technical Library (5)

7151 Technical Publications (1)

7613-2 Document Processing for DOE/OSTI (10)

8523-2 Central Technical Files (1)

2121 Pang-Chieh Chen (10) 

